\title{
4.9 ON THE STRUCTURE OF HYPERBOLIC INTERPLANETARY DUST STREAMS
}

\author{
I. Kresák and E.M. Pittich \\ Astronomical Institute \\ Slovak Academy of Sciences \\ Bratislava, Czechoslovakia
}

The only type of concentration of cometary dust with a reasonable probability of being detected by deep-space probes, are the dust tails emanating from passing comets. Essentially all the dust released from long-period comets leaves the solar system on hyperbolic orbits, because the radiation pressure limit is high (corresponding to centimetre-sized grains emitted in the vicinity of the Earth's orbit by comets of the oort's cloud). For the short-period comets the dynamical conditions for retention of emitted particles within the solar system are much more favourable, but those which remain in circumsolar orbits tend to disperse rather rapidly (Kresák, 1976a).

The Earth and its artificial satellites can encounter the dust debris only from comets whose perihelion distance is less than $1 \mathrm{~A} . \mathrm{U}$. On the average two such comets are observed per year. The average frequency of perihelion passages is $1.4 \mathrm{yr}^{-1}$ for long-period comets $(P>200 \mathrm{yr}), 0.1 \mathrm{yr}^{-1}$ for the intermediate group of the Comet Halley type $(20 \mathrm{yr}<\mathrm{P}<200 \mathrm{yr}), 0.4 \mathrm{yr}^{-1}$ for comets of the Jupiter family (4 $\mathrm{yr}<\mathrm{P}<20 \mathrm{yr}$ ), and $0.3 \mathrm{yr}^{-1}$ for comet Encke. The statistics of the long-period comets is less complete, but the bias is not particularly serious for $q<1$ (Kresák, 1975b). The short-period comets tend to liberate much less dust per revolution and their average absolute brightness is much lower. The probability of penetrating the tall of a shortperiod comet is increased by their concentration to the plane of ecliptic, but their low inclinations at the same time imply lower impact velocities and fluxes, and hence higher requirements on the minimum detectable concentration of particles. Taking all this into account it can be inferred that the prospects of detecting dust debris from comets are much better for nearly parabolic comets. 
The detectability of the direct dust tails has already been discussed, and predictions provided, by Poultney (1972, 1974). He concluded that each passage of a Pioneer $8 / 9$ detector would only be expected to yield one detected particle, but that other observable effects would be produced by the injection of the dust into the atmosphere.

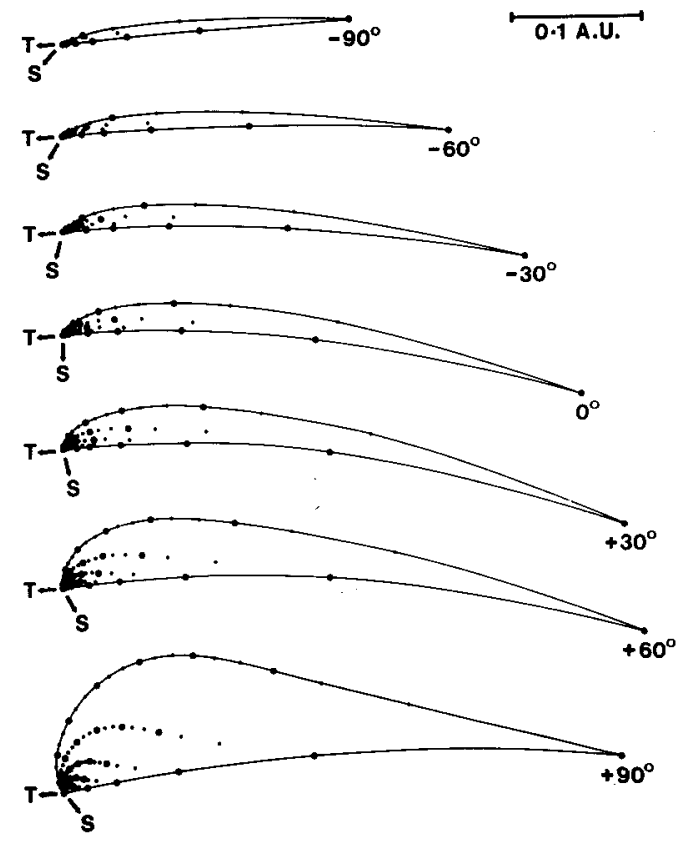

In order to get a clearer insight into the geometry and detectablifty of the dust tails, some model computations have been performed by the authors. The above figure shows the evolution of a dust stream produced by low-velocity emissions from a comet moving in a parabolic orbit of $q=0.5$. The dots indicate the crossing points of selected syndynes and synchrones. The upper boundary is the syndyne occupied by particles of mass $m=10^{-8} \mathrm{~g}$, assuming spherical shape and Whipple's (1967) mean density of photographic cometary meteors $\rho=0.448 \mathrm{~cm}^{-3}$. For Verniani's (1973) value $Q=0.8 \mathrm{~g} \mathrm{~cm}^{-3}$, obtained for faint radio meteors, this corresponds to $\mathrm{m}=3 \times 10^{-9} \mathrm{~g}$; for Ceplecha's (1967) value $Q=1.4 \mathrm{~g} \mathrm{~cm}^{-3}$ to $\mathrm{m}=10^{-9} \mathrm{~g}$; for stone of $\mathrm{Q}=3.2 \mathrm{~g} \mathrm{~cm}^{-3}$ to $\mathrm{m}=2 \mathrm{x}$ $10^{-10} \mathrm{~g}$; and for iron of $\rho=7.8 \mathrm{~g} \mathrm{~cm}^{-3}$ to $\mathrm{m}=3 \times 10^{-11} \mathrm{~g}$. Each following syndyne, proceeding downwards, refers to particle masses one order of magnitude higher, up to $10^{4}$ times the first value. The lower boundary is the synchrone formed by dust emission at true anomaly $\mathrm{v}=-150^{\circ}$, i.e. at a heliocentric distance of $7.5 \mathrm{AU}$. Proceeding to the left, the synchrone step is $+10^{\circ}$ in $\mathrm{v}$, with solid circles marking each 
$30^{\circ}$. If the amount of dust liberated from the nucleus is proportional to the radiative energy input, it is also proportional to $\mathrm{dv} / \mathrm{dt}$, and equal amounts are confined between each neighbouring pair of synchrones at a given time. If the mass distribution of the particles is an $\propto$ $\mathrm{m}^{-2} \mathrm{dm}$, a relation which is nearly satisfied for all permanent meteor showers in the visual range (Kresáková, 1966), each zone between two neighbouring syndynes includes an equal total mass, but a total number of particles differing by a factor of 10 increasing upwards. Instantaneous structures of the tail are plotted for seven values of $v(r \leq I)$, covering a period of 77.5 days; these are indicated on the right. The direction of motion of the comet $T$, defining the horizontal axis of the plot, and the direction to the Sun $s$ are also shown.

In spite of a number of simplifications involved, some interesting inferences are possible. Since the dust tail occupies a thin layer, the point of its crossing by the detector determines uniquely the size (syndyne) and time of release (symchrone) of the particles encountered. For this reason no information on the mass distribution or time dependence of the emissions can be gathered unless the probe moves in the orbiting plane of the comet. The curvature and width of the dust tail rather than its length tends to increase during the apparition. The area occupied by the dust increases faster than the mass input, so that for the post-perihelion arc the probability of encountering the tail is greater but, on the average, the flux is the same. The sunward boundary of the tail is determined by the heliocentric distance at which the comet begins to emit the dust particles; the outer boundary by the smallest particle mass which can be detected. Since the spread area varies nearly as $\mathrm{m}^{-2 / 3}$, the probability of encounter would tend to increase sharply with instrument sensitivity. On the other hand, if the thickness of the layer is proportional to the distance from the nucleus (due to the orthogonal component of the low emission velocities), the loci of uniform spatial concentration would nearly coincide with the synchrones in our model size distribution. An ingtrument with higher sensitivity for exploring the outer regions of the tail would detect relatively more sporadic particles which might mask the presence of the tail. Statistically, the encounter with the earlier emissions would be more likely. This implies that post-perihelion intercepts of the early pre-perihelion emissions, i.e. material from the time prior to a full development of the coma, has the highest probability of being recorded.

It should be emphasized that a single passage through a comet tail would not yield a reliable estimate of the total mass of the dust re- 
leased, since emissions in bursts may produce an irregular synchrone pattern. In general, the probability of detecting a dust stream is low and depends on the sensitivity of the instrument. Unless a probe is intentionally launched into a comet tail, only very small particles should be detectable a.t chance encounters, and the flux enhancement should mostly vanish in the sporadic background. A passage through the region of large particles and high particle concentrations would require a close approach to the comet and the crossing of its orbital plane at a suitable position angle, which would occur very rarely.

\section{REFERENCES}

Ceplecha, Z. (1967). In : Meteor Orbits and Dust, Smithson. Contrib. Astrophys. II = NASA SP-135, p. 35 .

Kresák, I. (1976a). This Volume.

Kresák, I. (1975b). Bull. Astron. Inst. Czech. 26, 92.

Kresáková, M. (1966). Contrib. Astron. Obs. Skalnaté Pleso 3, 75.

Poultney, S.K. (1972). Space Research 12, 403.

Poultney, S.K. (1974). Space Research 14, 707.

Verniani, F. (1973). J. Geophys. Res. 78, 8429.

Whipple, F.I. (1967). In : The Zodiacal Iight and the Interplanetary Medium. NASA SP-150, p. 409. 